\title{
ON SPECTRAL PROPERTIES OF ONE BOUNDARY VALUE PROBLEM WITH A SURFACE ENERGY DISSIPATION
}

\author{
O.A. ANDRONOVA, V.I. VOYTITSKY
}

\begin{abstract}
We study a spectral problem in a bounded domain $\Omega \subset \mathbb{R}^{m}$ depending on a bounded operator coefficient $Q>0$ and a dissipation parameter $\alpha>0$. In the general case we establish sufficient conditions ensuring that the problem has a discrete spectrum consisting of countably many isolated eigenvalues of finite multiplicity accumulating at infinity. We also establish the conditions, under which the system of root elements contains an Abel-Lidskii basis in the space $L_{2}(\Omega)$. In model one- and two-dimensional problems we establish the localization of the eigenvalues and find critical values of $\alpha$.
\end{abstract}

Keywords: spectral parameter, quadratic operator pencil, localization of eigenvalues, compact operator, Schatten-von-Neumann classes $S_{p}$, Abel-Lidskii basis property.

Mathematics Subject Classification: 35P05, 35P10

To the memory of Tomas Yakovlevich Azizov, whose results and lectures helped the authors in writing this work.

\section{INTRODUCTION}

In this work we study the spectral properties of one linear problem in mathematical physics depending on the dimension $m$ of a domain $\Omega \subset \mathbb{R}^{m}$ (with a piece-wise smooth boundary $\partial \Omega$ ), a bounded in $L_{2}(\Omega)$ operator coefficient $Q>0$ and a parameter $\alpha>0$ modeling the intensity of the energy dissipation on a part of the boundary $\Gamma$. Namely, we study the problem

$$
\begin{aligned}
-\Delta u & =-\lambda^{2} Q u & & (\text { in } \Omega), \\
\frac{\partial u}{\partial n} & =\alpha \lambda u & & (\text { on } \Gamma), \\
u & =0 & & (\text { on } S:=\partial \Omega \backslash \Gamma),
\end{aligned}
$$

for an unknown field $u=u(x)(x \in \Omega)$ and a spectral parameter $\lambda \in \mathbb{C}$.

This spectral problem is generated by the initial boundary value problem arising as a linearization of a nonlinear problem studied before in works by I.D. Chueshov [1]-[3]. Similar nonlinear problems can be found in the paper by J. Lagnese [4], where there was studied the damping of solutions to the wave equation in a bounded domain under the presence of a dissipation on the boundary as well as in work by I. Lasiecka и D. Tataru [5] devoted to the studying of an uniform stabilization of solutions on the boundary of a domain for a semi-linear wave equation with a nonlinear dissipation on the boundary.

It should be noted that in the one-dimensional case the known Regge problem has the same form. Such problem arises in the scattering theory. In the paper by A.A. Shkalikov [6], the Regge

O.A. Andronova, V.I. Voytitsky, On spectral properties of one boundary value problem WITH A SURFACE ENERGY DISSIPATION.

(c) Andronova O.A., Voytitsky V.I. 2017.

Submitted February 1, 2016. 
problem was reduced to the quadratic operator pencil with unbounded operator coefficients, the localization of the spectrum was established, estimates for the resolvent were obtained as well as the completenetss and the minimality of subsystems of root functions associated with a "half" of eigenvalues. Such approach seems to be suitable for studying problem (1)-(3). For instance, it was employed in paper by A.A. Shkalikov and A.V. Shkred [7] for studying a three-dimensional problem in the elasticity theory.

In this work, by means of the method of auxiliary boundary value problems of S.G. Krein kind, problem (1) $-(3)$ is reduced to some quadratic operator pencil in a general class described in the pioneer work by M.G. Krein and H. Langer [8] (see also [9]). However, general properties of the operator coefficients in this problem do not allow to apply known results on strongly or weakly damped pencils.

In the case $Q=I$ (the unit operator), this spectral problem as well as the corresponding initial boundary value problem were studied earlier by O.A. Andronova and N.D. Kopachevskii in [10], where they proved the discreteness of the spectrum and considered some model examples. In this work we obtain more general spectral results on existence of countably many isolated eigenvalues of finite multiplicity and the Abel-Lidskii basis property on the base of the theorems by T.Ya. Azizov, M.G. Krein-H. Langer, A.G. Kostyuchenko-M.B. Orazov, M.V. Keldysh and V.B. Lidskii. Moreover, in the work we describe more precisely the properties of a model twodimensional problem and establish the dynamics of the eigenvalues variation depending on the parameter $\alpha>0$. It turns out that each eigenvalue moves along a continuous curve in the right complex half-plane starting from the imaginary axis as $\alpha=0$ and returning back as $\alpha \rightarrow+\infty$. For some of the eigenvalues we find critical values of $\alpha$ corresponding to the beginning of moving to the imaginary axis.

We also note that the problems with a parameter in boundary conditions were studied earlier by many authors. In works by E.M. Russakovsky and A.A. Shkalikov (see [11] and [12]) there was constructed a general theory of ordinary differential equations of arbitrary order with a spectral parameter in a boundary condition and in the equation and there were established sufficient conditions for existence of the chains of eigenelements and adjoint elements forming a basis or a complete minimal system in the spaces of smooth functions. Rather general formulations of elliptic boundary value problems with a parameter linearly involved in boundary conditions were considered in works by J. Ercolano, M. Schechter, V.V. Barkovsky, A.N. Komarenko (see [13]-[15]). The asymptotics for the eigenvalues of such problems was studied in works by A.N. Kozhevnikov (see [16], [17]). Such problems arise in applications while studying hydrodynamical systems with free boundaries (see works by S.G. Krein and N.D. Kopachevsky), in diffraction theory (see works by M.S. Agranovich and co-authors) and others. In the present work we employ an abstract approach for studying boundary value problems and transmission problem in Lipschitz domains developed by S.G. Krein and N.D. Kopachevsky. It is based on using abstract and generalized Green's formula adapted for a particular problem (see works [18]-23]). In particular, such approach was used in works by the second co-author of the paper (see [21], [24], 25]) in studying some problems with the parameter linearly involved in an equation and boundary conditions.

\section{REDUCTION OF PROBLEM TO STUDYING QUADRATIC OPERATOR PENCIL}

In order to formulate problem (1)-(3) in operator form, we employ a generalized Green's formula for the Laplace operator and mixed boundary conditions, which arises as a particular case of the abstract Green's formula proved in works by N.D. Kopachevsky with co-authors [21]-[23]. In the last work the following result was provided. 
Theorem 2.1. Assume that the Lipschitz boundary $\partial \Omega$ of the domain $\Omega \subset \mathbb{R}^{m}$ is partitioned into pieces $\Gamma_{k}$ with Lipschitz boundaries $\partial \Gamma_{k}, k=\overline{1, q}$. Then following generalized Green's formula holds true for mixed boundary value problems:

$$
(\eta, u)_{H^{1}(\Omega)}=\langle\eta, u-\Delta u\rangle_{L_{2}(\Omega)}+\sum_{k=1}^{q}\left\langle\gamma_{k} \eta, \partial_{k} u\right\rangle_{L_{2}\left(\Gamma_{k}\right)}, \quad \forall \quad \eta, u \in \widehat{H}^{1}(\Omega) .
$$

Here the angle brackets stand for the value of the functionals determined by the elements $u-$ $\Delta u \in\left(\widehat{H}^{1}(\Omega)\right)^{*}$ and $\partial_{k} u:=\left(\frac{\partial u}{\partial n}\right)_{\Gamma_{k}} \in H^{-1 / 2}\left(\Gamma_{k}\right)$. At that, the trace operators $\gamma_{k} \eta:=\left.\eta\right|_{\Gamma_{k}}$ maps boundedly $H^{1}(\Omega)$ into $\widetilde{H}^{1 / 2}\left(\Gamma_{k}\right)$.

Remark 2.1. By $\widehat{H}^{1}(\Omega)$ we denote the subspace of the functions in $H^{1}(\Omega)$ such that each of the trace operators $\gamma_{k}$ maps boundedly them into the corresponding space $\widetilde{H}^{1 / 2}\left(\Gamma_{k}\right)$ of function defined on $\Gamma_{k}$ and extended by zero in the class $H^{1 / 2}(\Gamma)$ (see [23]).

If in the Sobolev space $H^{1}(\Omega)$ we introduce the equivalent norm

$$
\|u\|_{S}^{2}:=\int_{\Omega}|\nabla u|^{2} d \Omega+\int_{S}|u|^{2} d S
$$

and assume that $u \in H_{0, S}^{1}(\Omega):=\left\{u \in H^{1}(\Omega):\left.u\right|_{S}=0\right\}$, then the trace of such function on $\Gamma=\partial \Omega \backslash S$ will be extended by zero in the class $H^{1 / 2}(\partial \Omega)$. Hence, in view of Theorem 2.1 , we obtain the following result.

Corollary 2.1. Let the Lipschitz boundary $\partial \Omega$ of a domain $\Omega \subset \mathbb{R}^{m}$ is partitioned into two pieces $\Gamma$ and $S$ with Lipschitz boundaries. Then the generalized Green's formula

$$
(\eta, u)_{H_{0, S}^{1}(\Omega)}=\langle\eta,-\Delta u\rangle_{L_{2}(\Omega)}+\left\langle\gamma \eta, \frac{\partial u}{\partial n}\right\rangle_{L_{2}(\Gamma)}, \quad \forall \quad \eta, u \in H_{0, S}^{1}(\Omega)
$$

holds true, where $\gamma \eta:=\left.\eta\right|_{\Gamma}: H_{0, S}^{1}(\Omega) \rightarrow \widetilde{H}^{1 / 2}(\Gamma)$ is the operator of trace on $\Gamma$.

We shall seek the solutions to auxiliary boundary problems of S.G. Krein basing on formula (4).

First auxiliary problem (Newton problem for the Poisson equation): given a function $f$ defined in $\Omega$, find a solution $v \in H_{0, S}^{1}(\Omega)$ to the problem

$$
-\Delta v=f(\text { in } \Omega), \quad \frac{\partial v}{\partial n}=0(\text { on } \Gamma), \quad v=0(\text { on } S) .
$$

In accordance with formula (4), the classical solution should satisfy the identity

$$
(\eta, v)_{H_{0, S}^{1}(\Omega)}=\langle\eta, f\rangle_{L_{2}(\Omega)}, \quad \forall \eta \in H_{0, S}^{1}(\Omega) .
$$

As a weak solution of problem (4), we call each element $v \in H_{0, S}^{1}(\Omega)$ satisfying this identity for a fixed function $f$.

Definition 2.1. We shall say (see, for instance, [18, Sect. 3]) that two Hilbert spaces $F$ and $E$ form a Hilbert pair (we shall employ the notation $(F ; E)$ ) if $F$ is a dense linear subset in $E$ and there exists a bounded operator of embedding $F$ into $E$, that is, there exists a $>0$ such that

$$
\|u\|_{E} \leqslant a\|u\|_{F}, \quad \forall u \in F .
$$

At that, the generating operator of the Hilbert pair $(F ; E)$ is the operator $A: F \rightarrow F^{*}$ determined uniquely by the identity

$$
\langle\eta, A v\rangle_{E}=(\eta, v)_{F}, \quad \forall \eta, v \in F .
$$

It has the self-adjoint restriction $A: \mathcal{D}(A) \subset E \rightarrow E=\mathcal{R}(A)$ being a positive definite operator. 
Since the spaces $H_{0, S}^{1}(\Omega)$ and $L_{2}(\Omega)$ form a Hilbert pair of spaces and $H_{0, S}^{1}(\Omega)$ is compactly embedded into $L_{2}(\Omega)$, the following statement holds.

Lemma 2.1. For each $f \in\left(H_{0, S}^{1}(\Omega)\right)^{*}$ there exists a unique weak solution $v=A^{-1} f$ to problem (5). At that, the operator $A$ is the operator of the Hilbert pair $\left(H_{0, S}^{1}(\Omega) ; L_{2}(\Omega)\right)$. The restriction of this operator possessing the property $\mathcal{R}(A)=L_{2}(\Omega)$ is an unbounded positive definite operator in $L_{2}(\Omega)$. At that, $\mathcal{D}\left(A^{1 / 2}\right)=H_{0, S}^{1}(\Omega)$ and the identity

$$
\langle\eta, A v\rangle_{L_{2}(\Omega)}=(\eta, v)_{H_{0, S}^{1}(\Omega)}=\left(A^{1 / 2} \eta, A^{1 / 2} v\right)_{L_{2}(\Omega)}, \quad \forall \eta, v \in H_{0, S}^{1}(\Omega)
$$

holds. The operator $A: \mathcal{D}(A) \subset L_{2}(\Omega) \rightarrow L_{2}(\Omega)$ a positive discrete spectrum $\left\{\lambda_{k}(A)\right\}_{k=1}^{\infty}$ with the accumulation point $\lambda=+\infty$ and the asymptotic behavior (see [26])

$$
\lambda_{k}(A)=c_{A} k^{2 / m}[1+o(1)],(k \rightarrow \infty), c_{A}>0, m \geqslant 2 .
$$

The inverse operator $A^{-1}: L_{2}(\Omega) \rightarrow L_{2}(\Omega)$ is a compact positive operator.

Second auxiliary problem (the Newton problem for the Laplace equation): given a function $\psi$ defined on $\Gamma$, find a solution $w \in H_{0, S}^{1}(\Omega)$ to the problem

$$
-\Delta w=0(\text { in } \Omega), \quad \frac{\partial w}{\partial n}=\psi(\text { on } \Gamma), \quad w=0(\text { on } S) .
$$

In accordance with formula (4), a classical solution to this problem should satisfy the identity

$$
(\eta, w)_{H_{0, S}^{1}(\Omega)}=\langle\gamma \eta, \psi\rangle_{L_{2}(\Gamma)}, \quad \forall \eta \in H_{0, S}^{1}(\Omega) .
$$

As a weak solution of problem $(7)$, we shall call each element of $w \in H_{0, S}^{1}(\Omega)$ satisfying this identity for a fixed function $\psi$.

Since the trace operator $\gamma: H_{0, S}^{1}(\Omega) \rightarrow \widetilde{H}^{1 / 2}(\Gamma)$ is bounded (see [27]) for a fixed $\psi \in$ $\left(\widetilde{H}^{1 / 2}(\Gamma)\right)^{*}=H^{-1 / 2}(\Gamma)$, the expression $\langle\gamma \eta, \psi\rangle_{L_{2}(\Gamma)}$ is a bounded linear functional on the space $H_{0, S}^{1}(\Omega)$. Therefore, by the Riesz theorem, there exists a unique element $w=T \psi \in H_{0, S}^{1}(\Omega)$ such that

$$
\langle\gamma \eta, \psi\rangle_{L_{2}(\Gamma)}=(\eta, T \psi)_{H_{0, S}^{1}(\Omega)}, \quad \eta \in H_{0, S}^{1}(\Omega), \psi \in H^{-1 / 2}(\Gamma) .
$$

Thus, the bounded operator $T: H^{-1 / 2}(\Gamma) \rightarrow H_{0, S}^{1}(\Omega)$ is well-defined and it is adjoint to the operator $\gamma$ in the sense of identity (8). At that, $\mathcal{R}(T)=H_{0, S, h}^{1}(\Omega)$ is a subspace of harmonic functions in $H_{0, S}^{1}(\Omega)$. This leads us to the following result.

Lemma 2.2. For each $\psi \in H^{-1 / 2}(\Gamma)$ there exists a unique weak solution $w=T \psi \in H_{0, S, h}^{1}(\Omega)$ of problem (7).

The sum $u=v+w \in H_{0, S}^{1}(\Omega)$ of solutions to the first and second auxiliary problems is a weak solution of the problem

$$
-\Delta u=f(\text { in } \Omega), \quad \frac{\partial u}{\partial n}=\psi(\text { on } \Gamma), \quad u=0(\text { on } S) .
$$

This solution is generalized if $f \in L_{2}(\Omega), \psi \in L_{2}(\Gamma)$. The opposite statement holds as well (see an abstract result in [19]).

Lemma 2.3. Each element $u \in H_{0, S}^{1}(\Omega)$ can be uniquely represented as a sum of solutions to the first and second auxiliary problems, that is, as

$$
u=v+w=A^{-1} f+T \psi,
$$

where $f=-\Delta u \in\left(H_{0, S}^{1}(\Omega)\right)^{*}, \psi=\partial u / \partial n \in H^{-1 / 2}(\Gamma)$. 
This implies that the generalized eigenvalues and eigenfunctions of original problem (1) $-(3)$ satisfy the operator relation

$$
u=-\lambda^{2} A^{-1} Q u+\alpha T \gamma u, \quad u \in H_{0, S}^{1}(\Omega) .
$$

Making the change $\eta=A^{1 / 2} u \in L_{2}(\Omega)$ and applying the operator $A^{1 / 2}$ to the both sides of (11), we obtain the equivalent spectral problem for the pencil

$$
L_{\alpha}(\lambda) \eta:=\lambda^{2} A^{-1 / 2} Q A^{-1 / 2} \eta-\lambda \alpha\left(A^{1 / 2} T\right)\left(\gamma A^{-1 / 2}\right) \eta+\eta=0, \quad \eta \in L_{2}(\Omega) .
$$

By identity (8) and the compact embedding of $H^{1 / 2}(\Gamma)$ into $L_{2}(\Gamma)$ one can prove easily that the operators $\gamma A^{-1 / 2}: L_{2}(\Omega) \rightarrow L_{2}(\Gamma)$ and $A^{1 / 2} T: L_{2}(\Gamma) \rightarrow L_{2}(\Omega)$ are mutually adjoint and compact. Hence, the operator $\mathcal{B}:=\left(A^{1 / 2} T\right)\left(\gamma A^{-1 / 2}\right)$ is a negative compact operator in $L_{2}(\Omega)$ and it has an infinitely dimensional kernel $\operatorname{Ker} \mathcal{B}:=\left\{\eta \in L_{2}(\Omega): \eta=A^{1 / 2} u, u \in H_{0}^{1}(\Omega)\right\}$. If the domain is piece-wise smooth and its dimension satisfies the inequality $m \geqslant 2$, then the asymptotic formula (see [26])

$$
\lambda_{k}(\mathcal{B})=c_{\mathcal{B}} k^{-1 /(m-1)}[1+o(1)],(k \rightarrow \infty), c_{\mathcal{B}}>0,
$$

holds true.

The operator $\mathcal{A}:=A^{-1 / 2} Q A^{-1 / 2}$ is obviously compact positive operator in $L_{2}(\Omega)$. Employing the introduced notations, problem $(12)$ can be rewritten as

$$
L_{\alpha}(\lambda) \eta=\lambda^{2} \mathcal{A} \eta-\lambda \alpha \mathcal{B} \eta+\eta=0, \quad \eta \in L_{2}(\Omega) .
$$

\section{Simplest PROPERTIES OF SOLUTIONS TO SPECTRAL PROBLEMS}

Quadratic self-adjoint pencils of form (14) were studied before by many authors, the first results were presented in monograph [9]. However, the pencil with particular operators $\mathcal{A}$ and $\mathcal{B}$ is not in the class of problems described in [9], for which the localization of the eigenvalues was established and the theorems on completeness were proved.

The general properties of pencil (14) were established before in work [10]. We formulate them without proving.

$1^{0}$. The number $\lambda=0$ is not an eigenvalue of the problem.

$2^{0}$. All eigenvalues are located in the right complex half-plane symmetrically w.r.t. the real axis.

$3^{0}$. The spectrum of the problem can contain only eigenvalues of finite multiplicites $\left\{\lambda_{k}(\alpha)\right\}_{k=1}^{\infty}$ with the only possible accumulation point $\lambda=\infty$ (that is implied by the theorem by I.C. Gohberg valid for Fredholm operator pencils, see [9, Sect. 1.5]). At that, due to the compactness of the operator $\mathcal{B}$, the spectrum can contain only finitely many eigenvalues or no eigenvalues (see an example in [9, Sect. 5.12]).

$4^{0}$. As $\alpha=0$, the spectrum of the problem is located on the imaginary axis (hyperbolic case) and consists of infinitely many eigenvalues

$$
\left\{\lambda_{k}\right\}_{k=1}^{\infty}, \quad \lambda_{k}= \pm i \lambda_{k}^{-1 / 2}(\mathcal{A}), k=1,2, \ldots
$$

$5^{0}$. If $\alpha$ is formally equal to $\infty$, that is, we consider the limiting problem

$$
-\Delta u=-\lambda^{2} S u(\text { in } \Omega), \quad u=0(\text { on } \partial \Omega),
$$

then the spectrum is located on the imaginary axis and consists of infinitely many eigenvalues

$$
\left\{\lambda_{k}\right\}_{k=1}^{\infty}, \quad \lambda_{k}= \pm i \lambda_{k}^{-1 / 2}\left(\mathcal{A}_{0}\right), k=1,2, \ldots,
$$

where $\mathcal{A}_{0}:=A_{0}^{-1 / 2} Q A_{0}^{-1 / 2}$ is a compact positive operator in $L_{2}(\Omega), A_{0}$ is the positive defininte in $L_{2}(\Omega)$ operator of the Dirichlet problem for the Laplace equation. 
$6^{0}$. As $\alpha$ increases from zero, the eigenvalues $\lambda_{k}(\alpha)$ coinciding with numbers 15 as $\alpha=0$ shift to the right complex half plane perpendicular to the imaginary axis and as $\alpha \rightarrow+\infty$, they approach the limiting values (16) on the imaginary axis along perpendicular trajectories. This implies that for each $k$ there exists some critical value $\alpha_{k}^{*}>0$, after which $\lambda_{k}(\alpha)$ starts moving not from the imaginary axis but to this axis as $\alpha$ grows approaching the axis in the limit $\alpha=+\infty$. The considered below model examples will show that the critical values can be either independent of the eigenvalues or can depend on them but as $k \rightarrow \infty$, they tend to certain number. In the general case this fact is not proved.

\section{THEOREM ON DISCRETENESS OF SPECTRUM}

In problem 14 we make the change of the spectral parameter by the formula $\lambda=\mu^{-1}$ and consider instead the problem

$$
M_{\alpha}(\mu) \eta:=\mu^{2} \eta-\mu \alpha \mathcal{B} \eta+\mathcal{A} \eta=0 .
$$

In paper [10], there was given a proof for the following theorem by T.Ya. Azizov and this proof based essentially on a theorem by V.I. Matsaev on properties of non-self-adjoint Volterra operator, see [9, Sect. 4.11].

Theorem 4.1. Let

$$
\Phi(\mu):=\mu^{2} I+\mu B+C, \quad B=B^{*} \in \mathfrak{S}_{\infty}, 0 \leqslant C=C^{*} \in \mathfrak{S}_{\infty} .
$$

If at least one of the conditions

$$
B \in \mathfrak{S}_{p}, C^{1 / 2} \in \mathfrak{S}_{q} \backslash \mathfrak{S}_{p}(q>p>1)
$$

or

$$
B \in \mathfrak{S}_{p} \backslash \mathfrak{S}_{q}, C^{1 / 2} \in \mathfrak{S}_{q} \quad(1<q<p),
$$

holds true, then the pencil $\Phi(\mu)$ has countably many non-zero eigenvalues of finite multiplicities with the accumulation point $\lambda=0$.

We recall (see [9, Sect. 2.2]) that a compact (completely continuous) operator $A$ belongs to the Neumann-Schatten class $\mathfrak{S}_{p}(p>0)$ if its $s$-numbers, that is, the eigenvalues of the operators $\left(A^{*} A\right)^{1 / 2}$ are $p$-power summable, that is,

$$
\left.\sum_{j=1}^{\infty}\left(s_{j}(A)\right)^{p}=\sum_{j=1}^{\infty}\left(\lambda_{j}\left(A^{*} A\right)^{1 / 2}\right)\right)^{p}<\infty .
$$

It is obvious that if $A \in \mathfrak{S}_{p}$, then $A \in \mathfrak{S}_{p^{\prime}}$ for each $p^{\prime}>p$, and this is why it is reasonable to introduce the number $p_{*}:=\inf \left\{p \in \mathbb{R}: A \in \mathfrak{S}_{p}\right\}$.

Remark 4.1. Since the s-numbers of a compact non-negative operator $A$ coincides with its eigenvalues, the asymptotic formula $\lambda_{k}(A)=k^{-\beta}[1+o(1)],(k \rightarrow \infty)$ yields that $A \in \mathfrak{S}_{p}, p>$ $p_{*}=1 / \beta, A^{1 / 2} \in \mathfrak{S}_{p}, p>p_{*}=2 / \beta$.

It is known that for each bounded operator $B$, we have $A B, B A \in \mathfrak{S}_{p}$ if $A \in \mathfrak{S}_{p}$. It was proved in [28, Sect. 11.6] that under the condition $T_{i} \in \mathfrak{S}_{p_{i}}, 0<p_{i} \leqslant \infty, i=1$, 2, we have $T_{1} T_{2} \in \mathfrak{S}_{p}$, где $p^{-1}=p_{1}^{-1}+p_{2}^{-1}$.

Remark 4.2. By the induction one can establish a similar formula for the product of finitely many operators $T_{i}$, in particular, $T_{1} T_{2} T_{3} \in \mathfrak{S}_{p}$, where $p^{-1}=p_{1}^{-1}+p_{2}^{-1}+p_{3}^{-1}$.

We assume that $m \geqslant 2$. On the base of Remark 4.1 and asymptotic formula (13) we obtain that $\mathcal{B} \in \mathfrak{S}_{p}, p>p_{*}=m-1$. 
Lemma 4.1. If the operator $Q>0$ is boundedly invertible, then $\mathcal{A}^{1 / 2} \in \mathfrak{S}_{p}, p>p_{*}=m$.

Proof. Under the assumption of the lemma, there exist positive constants $c_{*}$ and $c^{*}$ such that $c_{*} I \leqslant Q \leqslant c^{*} I$. Hence,

$$
c_{*} A^{-1} \leqslant A^{-1 / 2} Q A^{-1 / 2} \leqslant c^{*} A^{-1}
$$

and therefore,

$$
c_{*} \lambda_{k}\left(A^{-1}\right) \leqslant \lambda_{k}(\mathcal{A}) \leqslant c_{*} \lambda_{k}\left(A^{-1}\right) .
$$

By asymptotic formula (6) we obtain that $A^{-1} \in \mathfrak{S}_{p}, p>p_{*}=m / 2$. Hence, employing the comparison tests for scalar series, we conclude that $\mathcal{A} \in \mathfrak{S}_{p}, p>p_{*}=m / 2$, and therefore, $\mathcal{A}^{1 / 2} \in \mathfrak{S}_{p}, p>p_{*}=m$.

Remark 4.3. For an arbitrary bounded $Q>0$ we have $\mathcal{A}^{1 / 2} \in \mathfrak{S}_{p}, p>m$.

Lemma 4.2. If $Q \in \mathfrak{S}_{r}, r>0$, then $\mathcal{A}^{1 / 2} \in \mathfrak{S}_{p}$, where $p \in\left(\frac{2 r m}{2 r+m} ; 2 r\right)$.

Proof. By formula (6) we have $A^{-1 / 2} \in \mathfrak{S}_{p^{\prime}}, p^{\prime}>p_{*}=m$. According to Remark 4.2, this implies that $\mathcal{A}=A^{-1 / 2} Q A^{-1 / 2} \in \mathfrak{S}_{p}$ for

$$
p=\left(\left(p^{\prime}\right)^{-1}+r^{-1}+\left(p^{\prime}\right)^{-1}\right)^{-1}=\left(\frac{2 r+p^{\prime}}{r p^{\prime}}\right)^{-1}=\frac{r p^{\prime}}{2 r+p^{\prime}} .
$$

Therefore, for each $p^{\prime}>m$ we have $\mathcal{A}^{1 / 2} \in \mathfrak{S}_{p}$ for $p=\left(2 r p^{\prime}\right) /\left(2 r+p^{\prime}\right)$. The image of the function $f\left(p^{\prime}\right):=\left(2 r p^{\prime}\right) /\left(2 r+p^{\prime}\right)$ on the set $(m ;+\infty)$ is the interval $((2 r m) /(2 r+m) ; 2 r)$.

Lemma 4.3. If $Q \in \mathfrak{S}_{r}$ for some $\frac{m-1}{2}<r<\frac{(m-1) m}{2}$, then $\mathcal{A}^{1 / 2} \in \mathfrak{S}_{m-1}$.

Proof. The statement is implied immediately from Lemma 4.2 since the assumptions of the lemma are equivalent to $m-1 \in\left(\frac{2 r m}{2 r+m} ; 2 r\right)$.

Theorem 4.2. If the operator $Q$ is boundedly invertible and $m \geqslant 2$ or $m=3$ and $S \in \mathfrak{S}_{r}$ for some $r \in(1 ; 3)$, then operator pencil $(17)$ has countably many non-zero eigenvalues of finite multiplicities with the accumulation point $\mu=0$.

Proof. We apply Theorem 4.1 with $B=-\alpha \mathcal{B}, C=\mathcal{A}$ to pencil 17 .

If the operator $Q$ is boundedly invertible, then by Lemma 4.1 we have $\mathcal{A}^{1 / 2} \in \mathfrak{S}_{p}, p>p_{*}=m$. Since earlier we established that $\mathcal{B} \in \mathfrak{S}_{p}, p>p_{*}=m-1$, then for each number $q \in(m-1 ; m)$ we have $\mathcal{B} \in \mathfrak{S}_{q}$ and at that, $\mathcal{A}^{1 / 2} \notin \mathfrak{S}_{q}$.

If $m=3$ and $S \in \mathfrak{S}_{r}, r \in(1 ; 3)$, then the assumptions of Lemma 4.3 are satisfied and by this lemma $\mathcal{A}^{1 / 2} \in \mathfrak{S}_{m-1}$. However, by the property $\mathcal{B} \in \mathfrak{S}_{p}, p>p_{*}=m-1$, we obtain that $\mathcal{B} \notin \mathfrak{S}_{m-1}$.

Corollary 4.1. Under the assumptions of the theorem, original pencil (14) (and (12)) has countably many eigenvalues of finite multiplicities with the accumulation point $\lambda=\infty$.

\section{THEOREMS ON COMPLETENESS OF ROOT FUNCTIONS}

We address now known results in the theory of self-adjoint quadratic operator pencils. One of the first results in this theory is the following theorem by M.G. Krein and H. Langer, see [8].

Theorem 5.1. Assume that we are given an operator function $L(\mu):=\mu^{2} I+\mu D+C$ acting in a Hilbert space $H$, where $D=D^{*} \in \mathcal{L}(H), C \geqslant 0, C \in \mathfrak{S}_{\infty}(H)$. Assume that its non-real 
spectrum formed by normal eigenvalues located symmetric w.r.t. the real axis is split into two parts $\sigma_{0}(L)=\Lambda \cup \bar{\Lambda}(\Lambda \cap \bar{\Lambda}=\emptyset)$. Then the operator equation

$$
L(Z):=Z^{2}+Z D+C=0
$$

has a solution $Z_{\Lambda}=K C^{1 / 2} \in \mathfrak{S}_{\infty}(H)$, where $K \in \mathcal{L}(H)$ is an angular operator. At that, the following properties hold:

1) $Z_{\Lambda}^{*} Z_{\Lambda} \leqslant C$

2) $\sigma_{0}\left(Z_{\Lambda}\right)=\Lambda$

3) for each $\mu \in \Lambda$ the operator $Z_{\Lambda}$ and the pencil $L(\mu)$ have the same Jordan chains.

The proof is based on applying the theory of operators acting in a space with indefinite metric. By the same method, A.G. Kostyuchenko and M.B. Orazov in [29] established a relation between the Jordan chains of the pencil and the operator root in the case of real eigenvalues. It was proved there that in the general case the entire discrete spectrum of the pencil $L(\mu)$ splits into the four parts

$$
\sigma_{d}(L)=F_{1} \cup \Lambda \cup F_{2} \cup \bar{\Lambda}
$$

where the sets $F_{i} \subset \mathbb{R}, i=1,2$, can have non-empty intersection and

$$
\sigma_{d}\left(Z_{\Lambda}\right)=F_{1} \cup \Lambda, \quad \sigma_{d}\left(Z_{\bar{\Lambda}}^{*}\right)=F_{2} \cup \bar{\Lambda}
$$

and $L(\mu)=\left(\mu I-Z_{\Lambda}\right)\left(\mu I-Z_{\bar{\Lambda}}^{*}\right)$. There was also described a procedure allowing one to select the parts of the Jordan chains of the pencil corresponding to $\mu \in F_{1}$, which are root functions for the operator $Z_{\Lambda}$. These results allows us to describe completely the spectral properties of the pencil $L(\mu)$ via the properties of the operators $Z_{\Lambda}$ and $Z_{\bar{\Lambda}}^{*}$. In particular, the completeness of the system of root functions of the operator $Z_{\Lambda}$ implies the completeness of the eigenelements and the adjoint elements of the pencil $L(\mu)$. We note that the completeness and the minimality of the root elements of the pencil $L(\mu)$ can be proved basing on the order and growth rate at the rays of the meromorphic function $L^{-1}(\mu)$, see paper by A.G. Kostyuchenko and A.A. Shkalikov [30]. However, in the present work this method is not employed.

In what follows we make use of the theorem by M.V. Keldysh and V.B. Lidskii proved in [31], see also [9]. Let $Z$ be a linear operator, $\widetilde{W}(Z)$ be the closure of the set $\{(Z u, u): u \in H\}$. It either coincides with an angle of opening $\theta_{Z} \leqslant \pi$ (with the vertex at the origin) or $\widetilde{W}(Z)=\mathbb{C}$.

Theorem 5.2. If for a compact operator $Z$ the set $\widetilde{W}(Z)$ is an angle of opening $\theta_{Z}=\pi / p$, where $p \geqslant 1$, and $Z \in \mathfrak{S}_{p}(H)$ (one can assume a weaker condition $s_{n}(Z)=o\left(n^{-1 / p}\right)(n \rightarrow \infty)$ ), then the system of root elements of the operator $Z$ is complete in the Hilbert space $H$.

In work [32] (se also [33] and [34, Ch. 5]), the following result was established.

Theorem 5.3. If under the assumptions of Theorem 5.2 the set $\widetilde{W}(Z)$ is an angle of openinig $\theta_{Z}<\pi / p$, where $p \geqslant 1 u Z \in \mathfrak{S}_{p}(H)$, then the system of root element $s$ of the operator $Z$ form a Fourier series in $H$ summable by the Abel method (in what follows we shall employ the notion of Abel-Lidskii basis of order $\beta>p$ ).

Remark 5.1. The definition of this summation method was first given by V.B. Lidskii in paper [32], some explanations can be found in recent works by M.S. Agranovich. For instance, in [34] the following definition was given. For a compact operator $A$ with a zero kernel and an image $\widetilde{W}(A)$ symmetric w.r.t. the number $\lambda_{0}:\left|\lambda_{0}\right|=1$, the property of being an Abel-Lidksii basis of order $\beta>0$ means that there exists a complete and minimal in the space $H$ system of root elements $\left\{f_{j}\right\}$ of the operator $A$ such that the formal series

$$
-\frac{1}{2 \pi i} \sum_{j} \oint_{\gamma_{j}} e^{-t \lambda_{0}^{-\beta} \lambda^{\beta}} A(I-\lambda A)^{-1} f d \lambda,
$$


where the contour $\gamma_{j}$ envelopes one isolated characteristic number of the operator A, converges after some arrangement of brackets independent of the element $f \in H$ to some function $f(t)$ and this function converges to $f$ as $t \rightarrow 0$.

It is obvious that the pencil $M_{\alpha}(\mu)$ satisfies the assumptions of Theorem 5.1 and at that, $Z=K \mathcal{A}^{1 / 2}$ (where $K$ is an angular operator) is contained in the same Neumann-Schatten class as the operator $\mathcal{A}^{1 / 2}$. In the general case, by the spectral properties of the problem, the set $\widetilde{W}(Z)$ is an angle of opening $\theta_{0} \leqslant \pi$. Assume that the set $\widetilde{W}(Z)$ is more narrow.

Theorem 5.4. The system of root functions of the pencil $M_{\alpha}(\mu)$ associated with the eigenvalues in the upper (or lower) complex plane as well as a part of root functions associated with the real eigenvalues form a complete system $L_{2}(\Omega)$ if $\theta_{0}<\frac{\pi}{m}$ or $\theta_{0}<\frac{\pi}{m}+\frac{\pi}{2 r}$ and $Q \in \mathfrak{S}_{r}$, $r>0$. At that, in the space $L_{2}(\Omega)$, this system form an Abel-Lidskii basis of order $\beta>m$ or $\beta>\beta_{0}:=\frac{2 r m}{2 r+m}$, respectively, in the first or second case.

Proof. It is obvious that for each $\theta_{0}<\pi / m$ we can find a small $\varepsilon>0$ such that $\theta_{0}<\pi /(m+\varepsilon)$. At that, it follows from Remark 4.3 that for each bounded $Q>0$ and each $\varepsilon>0$ we have $Z, \mathcal{A}^{1 / 2} \in \mathfrak{S}_{p}, p=m+\varepsilon$. Then by Theorem 5.3 the root elements of the operator $Z$ form an Abel-Lidskii basis of order $\beta>m+\varepsilon$. Since $\varepsilon$ is arbitrarily small, we can assume that $\beta>m$.

If $Q \in \mathfrak{S}_{r}, r>0$, by Lemma 4.2 we have $Z, \mathcal{A}^{1 / 2} \in \mathfrak{S}_{p}$, where $p \in\left(\beta_{0} ; 2 r\right)$. It follows from the condition $\theta_{0}<\pi / m+\pi /(2 r)=\pi / \beta_{0}$ that we can find a small $\varepsilon>0$ such that $\theta_{0}<\pi /\left(\beta_{0}+\varepsilon\right)$. Then by Theorem 5.3, the root elements of the operator $Z$ form Abel-Lidskii basis of order $\beta>\beta_{0}+\varepsilon$. Since $\varepsilon$ is arbitrarily small, it is sufficient to suppose that $\beta>\beta_{0}$.

Since each eigenelement and each adjoint element of the operator $Z$ is that for the pencil $M_{\alpha}(\mu)$, this completes the proof.

Corollary 5.1. If in addition we know that $\operatorname{Re} Z \geqslant 0$ and $\operatorname{Im} Z \geqslant 0$ or $\operatorname{Im} Z \leqslant 0$, then $\theta_{0} \leqslant \pi / 2$. It follows that the completeness holds if $m=2$ and $Q \in \mathfrak{S}_{r}, r>0$, or $m=3$ and $Q \in \mathfrak{S}_{r}, r<3$. This property likely holds for the pencil $M_{\alpha}(\mu)$ but the authors failed to prove it rigorously.

Remark 5.2. In Theorem 5.4 the conditions for the location of the image $\widetilde{W}(Z)$ in the angle of opening $\theta_{0}$ can be replaced by the condition on the existence of finitely many rays leaving the origin and partitioning the complex plane into sectors of opening less than $\theta_{0}$, on which the modified resolvent of the operator $Z$ obeys the estimate (see [33])

$$
\left\|Z(I-\lambda Z)^{-1}\right\|=O\left(|\lambda|^{-1}\right), \quad \lambda \rightarrow \infty .
$$

On the base of Theorem 5.4 and the fact that the transformation $\lambda=1 / \mu$ maps an angle of opening $\theta$ in the upper half-plane into an angle of the same opening in the lower half-plane (and vice versa), we obtain the following result.

Theorem 5.5 (Main theorem on properties of problem [1]-[3]). Under the assumptions of Theorem 5.4, the pencil $L_{\alpha}(\lambda)$ and original problem [1] [3] has a countable set $\sigma=\Lambda \cup \bar{\Lambda} \cup F$, $F \subset \mathbb{R}$ can be empty, $\Lambda \cap \mathbb{R}=\emptyset)$, of eigenvalues with the only accumulation point at infinity. At that, the root functions associated with the eigenvalues in the upper (lower) half-plane and a part of root functions corresponding to some subset $F_{1} \subset F$ (or $F_{2} \subset F$ ) form a complete system in $L_{2}(\Omega)$ being an Abel-Lidskii basis in this space.

Remark 5.3. Basing on the results by M.S. Agranovich in [26] (see also [35]), one can establish that the theorem is still true if in problem (1)-(3), the operator $-\Delta$ is replaced by a 
strongly elliptic formally self-adjoint second order differential system

$$
L \vec{u}:=-\sum_{i, j=1}^{m} \frac{\partial}{\partial x_{i}}\left(a_{i j}(x) \frac{\partial \vec{u}}{\partial x_{j}}\right)+c(x) \vec{u},
$$

and the normal derivative is replaced by the derivative along the conormal

$$
\partial_{\nu} \vec{u}:=\sum_{i, j=1}^{m} \nu_{i}(x) a_{i j}(x) \frac{\partial \vec{u}}{\partial x_{j}} .
$$

Here $\vec{u}=\left(u_{1}(x), \ldots, u_{n}(x)\right)(x \in \Omega)$ is the unknown vector function; $a_{i j}(x)$ are $n \times n$ matrices, whose entries obeys the symmetry condition $a_{i j}^{r s}(x)=a_{j i}^{s r}(x) ; c(x)$ is a symmetric positive definite matrix; $\nu=\left(\nu_{1}(x), \ldots, \nu_{m}(x)\right)$ is the unit outward normal vector for $\partial \Omega$. The principal symbol of the equations

$$
a(x, \xi):=\sum_{i, j=1}^{m} a_{i j}(x) \xi_{i} \xi_{j}, \quad \xi \in \mathbb{R}^{m},|\xi|=1,
$$

is a positive definite matrix uniformly in $x \in \bar{\Omega}$. Moreover,

$$
\sum a_{i j}^{r s}(x) \xi_{j}^{s} \xi_{i}^{r} \geqslant c \sum\left|\xi_{j}^{s}\right|^{2}, \quad x \in \Gamma, \quad \xi_{j}^{s} \in \mathbb{R}, \quad c>0 .
$$

\section{MODEL EXAMPLES}

Let us establish the localization for the eigenvalues of the problem in the case $S=I$ as $m=1$ or $m=2$ and $\Omega=(0 ; \pi) \times(0 ; 1)$.

We first consider the one-dimensional problem

$$
u^{\prime \prime}(y)-\lambda^{2} u(y)=0,0<y<1, \quad u(0)=0, \quad u^{\prime}(1)=\alpha \lambda u(1) .
$$

Earlier it was studied in [10], here we briefly provide their properties.

The corresponding characteristic equation is of the form

$$
\operatorname{coth} \lambda=\alpha, \quad 0 \leqslant \alpha<\infty .
$$

It is easy to confirm that as $\alpha=1$, the equation has no finite solutions, that is, the discrete spectrum is empty. If $\alpha \in(0 ; 1)$, we arrive at the sequence

$$
\lambda_{p}^{-}(\alpha):=c_{-}(\alpha)+i \pi(p-1 / 2), \quad c_{-}(\alpha):=\frac{1}{2} \ln \frac{1+\alpha}{1-\alpha}, \quad p= \pm 1, \pm 2, \ldots
$$

As $\alpha>1$, we have

$$
\lambda=\lambda_{p}^{+}(\alpha):=c_{+}(\alpha)+i \pi p, \quad c_{+}(\alpha):=\frac{1}{2} \ln \frac{\alpha+1}{\alpha-1}, \quad p=0, \pm 1, \pm 2, \ldots
$$

For a fixed $\alpha \neq 1$, the eigenvalues are located at the line $\operatorname{Re} \lambda=c_{-}(\alpha)$ or at the line $\operatorname{Re} \lambda=c_{+}(\alpha)$ parallel to the imaginary axis. As $\alpha$ varies, each eigenvalue moves along straight trajectories parallel the real axis and

$$
\lambda_{p}^{-} \rightarrow \infty(\alpha \rightarrow 1-0), \quad \lambda_{p}^{+}(\alpha) \rightarrow \infty(\alpha \rightarrow 1+0) .
$$

Thus, $\alpha=1$ is the critical value of the parameter for all eigenvalues. As $0<\alpha<1$, all eigenvalues of problem (23) are non-real, as $\alpha>1$ there is one positive eigenvalue $\lambda_{0}^{+}(\alpha)$, which moves to the left as $\alpha$ grows and tends to zero as $\alpha \rightarrow+\infty$. For $p \neq 0$, the eigenvalues $\lambda_{p}^{+}(\alpha)$ tend to the numbers $\lambda_{p}^{+}(+\infty)=i \pi p$ as $\alpha \rightarrow+\infty$ and these numbers are the solutions to the Dirichlet spectral problem:

$$
u^{\prime \prime}(y)-\lambda^{2} u(y)=0, \quad 0<y<1, \quad u(0)=u(1)=0 .
$$


Now we consider the two-dimensional spectral problem

$$
\left.\Delta u-\lambda^{2} u=0(\text { in } \Omega), \quad u=0(\text { on } S), \quad \frac{\partial u}{\partial y}-\lambda \alpha u=0 \text { (on } \Gamma\right),
$$

in the domain $\Omega=(0 ; \pi) \times(0 ; 1)$, where $\Gamma:=\{(x ; 1): x \in(0 ; 1)\}$.

We seek the solutions as

$$
u(x, y)=u_{n}(x, y)=\sin (n x) Y_{n}(y), n=0,1,2, \ldots,
$$

and for each $n$ we obtain the one-dimensional problem

$$
Y_{n}^{\prime \prime}-\left(\lambda^{2}+n^{2}\right) Y_{n}=0,0<y<1, \quad Y_{n}(0)=0, \quad Y_{n}^{\prime}(1)=\lambda \alpha Y_{n}(1) .
$$

It has the solution

$$
Y_{n}(y)=\sinh \left(y \sqrt{\lambda^{2}+n^{2}}\right)
$$

which in view of the last boundary condition implies the series of characteristic equations

$$
\operatorname{coth} \sqrt{\lambda^{2}+n^{2}}=\frac{\alpha \lambda}{\sqrt{\lambda^{2}+n^{2}}}, \quad n=1,2, \ldots, \quad \operatorname{Re} \sqrt{\lambda^{2}+n^{2}} \geqslant 0 .
$$

We observe that as $n=0$, we obtain characteristic equation (24) of the one-dimensional spectral problem but for $n>0$ its properties differ substantially.

If we make the change $z=\lambda^{2}+n^{2}$, by employing formulae for hyperbolic functions we obtain the equivalent equation

$$
f(z):=\cosh (2 \sqrt{z})\left[\left(\alpha^{2}-1\right) z-\alpha^{2} n^{2}\right]-\left[\left(\alpha^{2}+1\right) z-\alpha^{2} n^{2}\right]=0 .
$$

The function $f(z)$ is entire and by Picard's little theorem (see, for instance, [36, Sect. 8.8]), the equation $f(z)=A$ always has infinitely many roots with the accumulation point at infinity except probably one exceptional value of the constant $A$. Since the order of the function $\cosh (2 \sqrt{z})$ is equal to $1 / 2$, the function $f(z)$ has the same order if $\left(\alpha^{2}-1\right) z-\alpha^{2} n^{2} \not \equiv 0$. The latter is possible only if $\alpha=1, n=0$. Thus, as $n>0$, the function $f(z)$ has a fractional order and therefore, there are no exceptional values of $A$ and equation (33) has infinitely many solutions $\lambda_{n k}$. This is confirmed by Theorem 5.4.

For a fixed $n>0$ and $\alpha \neq 1$, we have

$$
\frac{\left(\alpha^{2}+1\right) z-\alpha^{2} n^{2}}{\left(\alpha^{2}-1\right) z-\alpha^{2} n^{2}} \rightarrow \frac{\alpha^{2}+1}{\alpha^{2}-1}
$$

as $z \rightarrow \infty$. Therefore, as $z \rightarrow \infty$, the roots of the equation 33 converge to the roots of the same equation as $n=0$, that is, to numbers (25) or (26). A simple analysis shows that as $\alpha<1$, equation (33) has no real eigenvalues, for $\alpha>1$ the equation has the unique positive root converging to zero as $\alpha \rightarrow \infty$. By Theorem 5.5, as $\alpha<1$, the system of the root functions associated with the eigenvalues in the upper or lower half-plane is complete in the space $L_{2}(\Omega)$ if $\theta_{0}<\pi / 2$. As $\alpha=1$, the completeness seems to be lost since as $n=0$, infinitely many eigenvalues $\lambda_{0 k}$ pass to infinity (in this case it is obvious that $\theta_{0} \geqslant \pi / 2$ ).

If $\alpha=1, n>0$, making the change $\zeta=\sqrt{z}$ in equation $(33)$, we obtain

$$
\sinh \zeta= \pm i \frac{\zeta}{n}
$$

For each fixed $n>0$ this equation has infinitely many solutions

$$
\zeta_{n k}=v_{n k} \pm i w_{n k} \rightarrow \infty, \quad k \rightarrow \infty .
$$

It is easy to confirm that in this case we necessarily have $v_{n k} \rightarrow+\infty$. Hence, for large $k$ this equation is close to the equation $e^{\zeta}= \pm 2 i \frac{\zeta}{n}$. Therefore, $e^{2 v}=\frac{4}{n^{2}}\left(v^{2}+w^{2}\right)$ and as $v \rightarrow+\infty$, we obtain that $|w| \rightarrow \frac{n}{2} e^{v}$. It is easy to prove that $w_{n k}= \pm \pi k+o(1)(k \rightarrow \infty)$ and therefore, 
$v_{n k}=\ln \frac{2 \pi k}{n}+o(1)$. Since as $k \rightarrow \infty$, the eigenvalues satisfy $\lambda_{n k}=\sqrt{\zeta_{n k}^{2}-n^{2}} \rightarrow \zeta_{n k}$, then $\lambda_{n k} \rightarrow \ln \frac{2 \pi k}{n} \pm i \pi k$. Therefore, in distinction to $\alpha \neq 1$, as $\alpha=1$ and $k \rightarrow \infty$, the root converge not along the lines parallel to the imaginary axis but along the exponential curves $f_{ \pm}(x)= \pm \frac{n}{2} e^{x}$.

For a fixed $n$, as $\alpha$ grows, the eigenvalues $\lambda_{n k}=x+i y$ move in the complex plane along countably many continuous curves, each of them is described by the implicit function

$$
\operatorname{Im}\left(\frac{\sqrt{(x+i y)^{2}+n^{2}}}{(x+i y)} \operatorname{coth} \sqrt{(x+i y)^{2}+n^{2}}\right)=\operatorname{Im} \alpha=0 .
$$

Each of this curves starts and ends at the imaginary axis attaining the maximal value for some $\alpha_{n k}^{*}>0$. The results of numerical calculations (up to $10^{-4}$ ) are presented in the table.

\begin{tabular}{|c|c|c|c|c|c|c|c|c|}
\hline \multicolumn{3}{|c|}{$n=1$} & & \multicolumn{4}{c|}{$n=2$} \\
\hline$k$ & $\alpha_{n k}^{*}$ & $x_{n k}^{\text {max }}$ & $y_{n k}$ & & $k$ & $\alpha_{n k}^{*}$ & $x_{n k}^{\text {max }}$ & $y_{n k}$ \\
\hline 1 & 0.9984 & 1.7545 & 2.6480 & & 1 & 0.9360 & 1.0270 & 3.2199 \\
\hline 2 & 0.9952 & 2.6051 & 5.7417 & & 2 & 0.9689 & 1.9301 & 5.9614 \\
\hline 3 & 0.9962 & 3.1242 & 8.7794 & & 3 & 0.9829 & 2.4716 & 8.9615 \\
\hline$\ldots$ & $\ldots$ & $\ldots$ & $\ldots$ & & $\ldots$ & $\ldots$ & $\ldots$ & $\ldots$ \\
\hline 10 & 0.9995 & 4.7222 & 30.6147 & & 10 & 0.9980 & 4.0925 & 30.7085 \\
\hline
\end{tabular}

\begin{tabular}{|c|c|c|c|c|c|c|c|c|}
\hline \multicolumn{3}{|c|}{$n=3$} & & \multicolumn{4}{c|}{$n=10$} \\
\hline$k$ & $\alpha_{n k}^{*}$ & $x_{n k}^{\text {max }}$ & $y_{n k}$ & & $k$ & $\alpha_{n k}^{*}$ & $x_{n k}^{\text {max }}$ & $y_{n k}$ \\
\hline 1 & 0.8102 & 0.7154 & 3.9163 & & 1 & 0.3271 & 0.2228 & 10.3107 \\
\hline 2 & 0.9198 & 1.5447 & 6.3354 & & 2 & 0.5378 & 0.6095 & 11.4494 \\
\hline 3 & 0.9570 & 2.0817 & 9.1860 & & 3 & 0.6839 & 0.9967 & 13.2420 \\
\hline$\ldots$ & $\ldots$ & $\ldots$ & $\ldots$ & & $\ldots$ & $\ldots$ & $\ldots$ & $\ldots$ \\
\hline 10 & 0.9955 & 3.7235 & 30.8129 & & 10 & 0.9520 & 2.5985 & 32.2284 \\
\hline
\end{tabular}

By the table we see that the critical values $\alpha_{n k}^{*}$ depend essentially on the indices $n$ and $k$. For a fixed $n>0$ and $k \rightarrow \infty$ we likely have $\alpha_{n k}^{*} \rightarrow 1-0$ and hence, $x_{n k}^{\max } \rightarrow \ln \frac{2 \pi k}{n}, y_{n k} \rightarrow \pi k$. For a fixed $k$, as $n$ grows, the values $\alpha_{n k}^{*}$ and associated $x_{n k}^{\max }$ decrease monotonically and at that, $y_{n k}$ increase monotonically. For a fixed $\alpha$ and $k$, the eigenvalues $\lambda_{n k}$ behave in the same way. This is due to the fact that as $n$ grows, functions 36 tend to the imaginary axis moving away from the real axis.

The authors thank N.D. Kopachevsky for useful discussions and advices.

\section{BIBLIOGRAPHY}

1. I.D. Chueshov. Introduction into the theory of infinite-dimensional dissipative systems. Akta, Kharkov (2006). (in Russian).

2. I. Chueshov, M. Eller, I. Lasiecka Finite dimensionality of the attractor for a semilinear wave equation with nonlinear boundary dissipation // Comm. Part. Diff. Equat. 29:11-12, 1847-1876 (2004).

3. I. Chueshov, I. Lasiecka. Global attractors for von karman evolutions with a nonlinear boundary dissipations // J. Diff. Equat. 198:1, 196-231 (2004).

4. J. Lagnese. Decay of the solution of the wave equation in a bounded region with boundary dissipation // J. Diff. Equat. 50:2, 163-182 (1983). 
5. I. Lasiecka, D. Tataru. Uniform boundary stabilization of semilinear wave equation with nonlinear boundary dissipation // Diff. Integ. Equat. 6:3, 507-533 (1993).

6. A.A. Shkalikov. Spectral analysis of the Regge problem // Russ. J. Math. Phys. Vol. 8:3, 356-364 (2001).

7. A.A. Shkalikov, A.V. Shkred. The problem of steady-state oscillations of a transversally isotropic half-cylinder // Matem. Sborn. 182:8, 1222-1246 (1991).

8. M.G. Krein, H. Langer. On some mathematical principles of the theory on damped oscillations of continua // Proc. of Intern. Simposium on applying theory of functions of complex variable in the continuum mechanics. Nauka, Moscow, 283-322 (1965). (in Russian).

9. I.C. Gohberg, M.G. Krein. Introduction to the theory of linear nonselfadjoint operators. Nauka, Moscow (1965). [Trans. Math. Monogr. 18. Amer. Math. Soc. Providence, RI. (1969).]

10. O.A. Andronova, N.D. Kopachevskii. On linear problems with surface dissipation of energy // Sovr. Matem. Fundamen. Napr. 29, 11-28 (2008). [J. Math. Sci. 164:4, 478-496 (2010).]

11. E.M. Russakovskii. Operator treatment of boundary problems with spectral parameters entering via polynomials in the boundary conditions // Funkts. Anal. Pril. 9:4, 91-92 (1975). [Funct. Anal. Appl. 9:4, 358-359 (1975).]

12. A.A. Shkalikov. Boundary value problems for ordinary differential equations with a parameter in the boundary conditions // Tr. Semin. Im. I.G. Petrovskogo 9, 190-229 (1983).

13. J. Ercolano, M. Schechter. Spectral theory for operators generated by elliptic boundary problems with eigenvalue parameter in boundary conditions I-II // Comm. Pure Appl. Math. 18:1-2, 83-105 (1965).

14. V.V. Barkovskii. Expansion over eigenfunctions of self-adjoint operators corresponding to general elliptic problems with eigenvalue in boundary conditions // Ukrain. Matem. Zhurn. 19:1, 9-24 (1967). (in Russian).

15. O.N. Komarenko. Expansions over eigenvectors of self-adjoint operators generated by general transmission problem // Collect. works of Inst. Math. NAS Ukraine. 2:1, 127-157 (2005). (in Ukrainian).

16. A.N. Kozhevnikov. On the asymptotics of eigenvalues and on completeness of the root vectors of an operator generated by a boundary value problem with a spectral parameter in a boundary condition // DAN SSSR. 200:6, 1273-1276. [Sov. Math. Dokl. 12, 1564-1568 (1971).]

17. A.N. Kozhevnikov. Spectral problems for pseudodifferential systems elliptic in the DouglisIINirenberg sense, and their applications // Matem. Sborn. 92(134):1(9), 60-88 (1973). [Math. USSRSbornik. 21:1, 63-90 (1973).]

18. N.D. Kopachevskiǐ, S.G. Krě̌n, Ngo Zuj Kan. Operator methods in hydrodynamics. Evolutionary and spectral problems. Nauka, Moscow (1989).

19. N.D. Kopachevskii. On abstract Green formula for a triple of Hilbert spaces and its applications to the Stokes problem // Tavricheskii Vestn. Inform. Matem. 2, 52-80 (2004). (in Russian).

20. N.D. Kopachevskii. Abstract Green formula for mixed boundary value problems // Uchenye Zapis. Tavricheskogo Natsional. Univ. im V.I. Vernadskogo. Ser. Matem. Mekh. Inform. Kibern. 20(59):2, 3-12 (2007). (in Russian).

21. V.I. Voititskiy, N.D. Kopachevskiy, P.A. Starkov. Multicomponent conjugation problems and auxiliary abstract boundary-value problems // Sovr. Matem. Fundamen. Napr. 34, 5-44 (2009). [J. Math. Sci. 170:2, 131-172 (2010).]

22. N.D. Kopachevskii, K.A. Radomirskaya. Abstrat mixed boundary value problems and spectral matching problems // Uchenye Zapis. Tavricheskogo Natsional. Univ. im V.I. Vernadskogo. Ser. Matem. Mekh. Inform. Kibern. 27(66):1, 58-64 (2014). (in Russian).

23. N.D. Kopachevskii. On abstract Green formula for a triple of Hilbert spaces and sesquilinear forms // Sovr. Matem. Fundamen. Napr. 57, 71-107 (2015). (in Russian).

24. V.I. Voytitsky. On spectral problems generated by linear Stefan problelm with Gibbs-Thompson condition // Nelin. Granich. Zadachi. 17, 31-49 (2007). (in Russian).

25. V. Voytitsky. On some class of self-adjoint boundary value problems with the spectral parameter in the equations and the boundary conditions // Operator Theory: Advan. Appl. Springer, Basel. 221, 635-651. (2012). 
26. M.S. Agranovich. Spectral problems for second-order strongly elliptic systems in smooth and nonsmooth domains // Uspekhi Matem. Nauk. 57:5(347), 3-78 (2002). [Russ. Math. Surv. 57:5, 847-920 (2002).]

27. E. Gagliardo. Caratterizzazioni delle trace sulla frontiera relative ad alaine classi di funzioni in $n$ variabili // Rendiconti del Seminare Matematico della Universita di Padova. 27, 284-305 (1957). (In Italian).

28. M.Sh. Birman, M.Z. Solomyak. Spectral theory of self-adjoint operators in Hilbert space. Lan', St.-Petersburg (2010). (in Russian).

29. A.G. Kostyuchenko, M.B. Orazov. Certain properties of the roots of a self-adjoint quadratic pencil // Funkts. Anal. Pril. 9:4, 28-40 (1975). [Funct. Anal. Appl. 9:4, 295-305 (1975)]

30. A.G. Kostyuchenko, A.A. Shkalikov. Self-adjoint quadratic operator pencils and elliptic problems // Funkts. Anal. Pril. 17:2, 38-61 (1983). [Funct. Anal. Appl. 17:2, 109-128 (1983).]

31. M.V. Keldysh, V.B. Lidskii. Issues in spectral theory of non-self-adjoint operators // Proc. IV All-Union Matem. Congress. 1, 101-120 (1963). (in Russian).

32. V.B. Lidskii. Summability of series in terms of the principal vectors of non-selfadjoint operators // Trudy Mosk. Matem. Obsc. 3-35 (1962). (in Russian).

33. A.S. Markus. Certain criteria for the completeness of a system of root-vectors of a linear operator in a Banach space // Matem. Sborn. 70:4, 526-561 (1966). (in Russian).

34. M.S. Agranovich, B.Z. Katsenelenbaum, A.N. Sivov, N.N. Voitovich. Generalized method of egenoscillations in difraction theory. Viley-VCH, Berlin (1999).

35. M.S. Agranovich. Spectral problems in Lipschitz domains // Sovr. Napr. Fundamen. Matem. 11-35 (2011). [J. Math. Sci. 190:1, 8-33 (2013)]

36. A.I. Markushevich. Theory of analytic functions. V. 2. Nauka, Moscow (1968). (in Russian).

Ol'ga Andreevna Andronova,

Academy of Construction and Architecture,

V.I. Vernadsky Crimean Federal University,

Kievskaya str. 181,

295493, Simferopol, Republic of Crimea, Russia

E-mail: o.andronova@list.ru

Viktor Ivanovich Voytitsky,

Taurida Academy,

V.I. Vernadsky Crimean Federal University,

Acad. V.I. Vernadsky av. 4,

295007, Simferopol, Republic of Crimea, Russia

E-mail: victor.voytitsky@gmail.com 